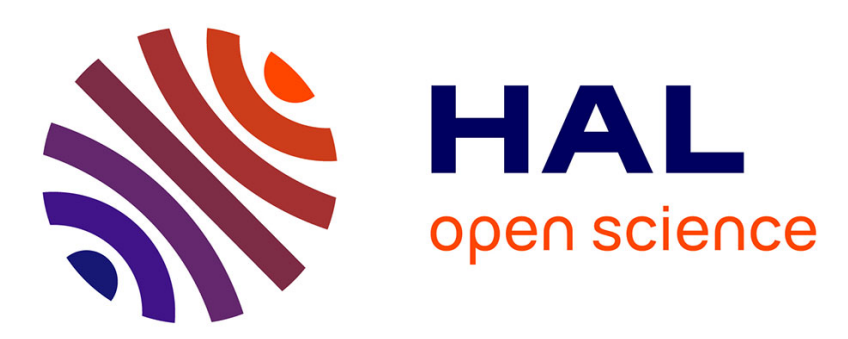

\title{
Moyens de diagnostic pour l'étude spatio-temporelle de l'émission X d'un plasma créé par laser
}

\author{
J.P. Gex, N. Fleurot, R. Sauneuf
}

\section{To cite this version:}

J.P. Gex, N. Fleurot, R. Sauneuf. Moyens de diagnostic pour l'étude spatio-temporelle de l'émission X d'un plasma créé par laser. Revue de Physique Appliquée, 1977, 12 (7), pp.1049-1053. 10.1051/rphysap:019770012070104900 . jpa-00244276

\section{HAL Id: jpa-00244276 https://hal.science/jpa-00244276}

Submitted on 1 Jan 1977

HAL is a multi-disciplinary open access archive for the deposit and dissemination of scientific research documents, whether they are published or not. The documents may come from teaching and research institutions in France or abroad, or from public or private research centers.
L'archive ouverte pluridisciplinaire HAL, est destinée au dépôt et à la diffusion de documents scientifiques de niveau recherche, publiés ou non, émanant des établissements d'enseignement et de recherche français ou étrangers, des laboratoires publics ou privés. 


\author{
Classification \\ Physics Abstracts \\ $6.570-0.692-2.650$

\section{MOYENS DE DIAGNOSTIC POUR L'ÉTUDE SPATIO-TEMPORELLE (*) DE L'ÉMISSION X D'UN PLASMA CRÉÉ PAR LASER}

\author{
J. P. GEX, N. FLEUROT et R. SAUNEUF \\ Commissariat à l'Energie Atomique, Centre d'Etudes de Limeil \\ B. P. No 27, 94190 Villeneuve St Georges, France
}

(Reçu le 30 décembre 1976, accepté le 29 mars 1977)

\begin{abstract}
Résumé. - Le développement des lasers de puissance délivrant des impulsions subnanosecondes demande l'élaboration de diagnostics permettant de mesurer les effets de leur interaction avec la matière. Les possibilités, à moyen terme, de comprimer de manière significative des cibles de petit diamètre $(<500 \mu \mathrm{m})$ nécessitent d'explorer la gamme de temps qui va de quelques picosecondes à quelques nanosecondes, avec des résolutions spatiales pouvant atteindre quelques microns dans le domaine spectral compris entre 1 et $10 \mathrm{keV}$.

De tels diagnostics sont actuellement possibles grâce à la mise au point de microscope $X$ à très haute résolution spatiale $(2$ à $3 \mu \mathrm{m})$ et de caméras $X$ à balayage de fente à haute résolution temporelle ( $<50 \mathrm{ps})$.

Nous présentons les performances obtenues avec de tels dispositifs ainsi que des exemples d'applications.

Abstract. - The development of subnanosecond high intensity lasers requires diagnoses to measure the effects of their interaction with matter. In order to characterize the compression of small targets (diameter $<500 \mu \mathrm{m}$ ) it is necessary to have X-ray devices with a temporal resolution of $10 \mathrm{ps}$ and a spatial resolution of a few microns within the range of 1 to $10 \mathrm{keV}$.

The use of X-ray microscopes having a spatial resolution of 2-3 $\mu \mathrm{m}$, of streak cameras with time resolution $<50$ ps and of framing camera tubes of 200 ps framing time allows such diagnoses.

Here, we discuss the performance of these devices and some applications.
\end{abstract}

1. Introduction. - L'un des diagnostics importants pratiqué autour des expériences d'interaction lasermatière est d'observer le comportement spatio ou spectro-temporel de l'émission $\mathrm{X}$ du plasma $[1,2]$.

A cette fin il faut pouvoir disposer d'optiques et de systèmes d'enregistrement qui couvrent le domaine spectral compris entre 1 et $10 \mathrm{keV}$ tout en permettant d'obtenir :

- une résolution spatiale au niveau de l'objet de quelques microns à dix microns (soit environ 100 fois inférieure à la dimension de la cible) ;

- une résolution temporelle de quelques picosecondes à une centaine de picosecondes (soit environ 10 fois inférieure à la durée de l'impulsion laser).

La méthode de mesure est schématisée par la figure 1. Un objectif $\mathrm{X}$ (chambre à sténopé ou microscope $\mathrm{X}$ à

$\left(^{*}\right)$ Communication présentée au Congrès National de Physique des Plasmas, Paris 6-10 décembre (1976). miroirs) à haut pouvoir résolvant forme l'image agrandie du plasma sur la photocathode d'une caméra électronique à haute résolution temporelle (caméra à balayage de fente ou tube obturateur).

Notre propos sera de présenter successivement les performances actuelles de chacun de ces éléments tout en indiquant certaines de leurs limitations.

2. Optiques X. - Deux critères doivent être respectés simultanément : haute résolution spatiale et grande ouverture ; en effet une diminution des temps de pose au niveau des caméras électroniques implique un accroissement correspondant de l'éclairement de leur photocathode ; par ailleurs, les optiques doivent travailler à grandissement élevé (20 à 100) afin de rendre compatible la résolution spatiale intrinsèque des caméras $(100$ à $150 \mu \mathrm{m})$ avec celle désirée au niveau de l'objet ( 1 à $5 \mu \mathrm{m}$ ).

La caméra à sténopé satisfait imparfaitement à ces deux critères : si la résolution spatiale (dans la 


\section{OPTIQUES}
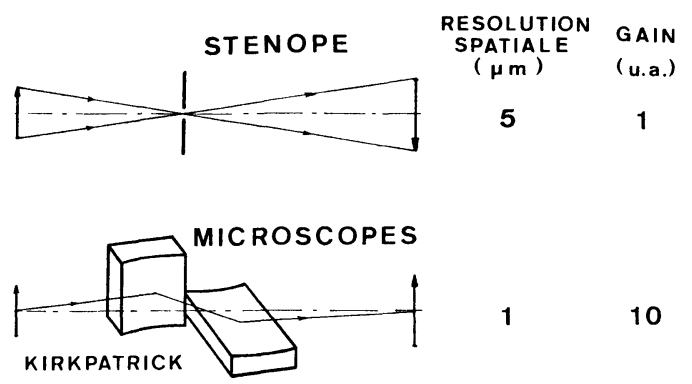

10

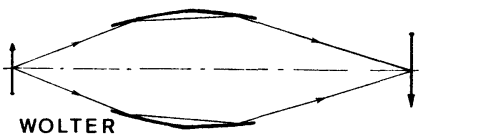

1000

\section{CAMERAS}

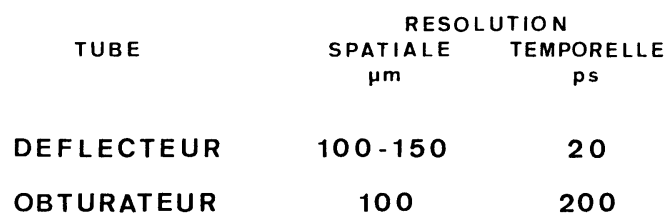

Fig. 1. - Méthodes de mesure de l'émission $\mathbf{X}$ d'un plasma [Principle of X-ray diagnoses.]

limite géométrique) croît linéairement lorsque le diamètre du trou décroît, inversement l'éclairement image décroît paraboliquement.

Pratiquement on ne peut guère espérer une résolution spatiale meilleure que $5 \mu \mathrm{m}$.

Dans ces conditions nous avons pensé utiliser, comme d'autres expérimentateurs étrangers $[3,4]$ des microscopes $\mathrm{X}$ étudiés il y a déjà une dizaine d'années par Kirkpatrick et Wolter [5, 6].

En effet, ces systèmes qui utilisent la propriété de réflexion totale des rayons $X$ sous incidence rasante satisfont non seulement aux deux critères déjà évoqués mais jouent également le rôle de filtre passe-bas en énergie ; leur résolution spatiale est de l'ordre du micron et leur ouverture est 10 à 1000 fois plus élevée que celle d'une chambre à sténopé (10 fois dans le cas des microscopes à 2 miroirs cylindriques croisés dits de type Kirkpatrick, 1000 fois dans le cas des microscopes composés d'un hyperboloïde et d'un ellipsoïde de révolution coaxiaux dits de type Wolter) (Fig. 1).

Dans un premier temps nous avons réalisé des microscopes simples de type Kirkpatrick (Fig. 2).

L'examen des images obtenues montre qu'en utilisant des miroirs cylindriques en verre ou en or, on obtient sur l'objet une résolution spatiale meilleure que $2 \mu \mathrm{m}$. A titre d'exemple, la figure 3 présente le densitogramme de l'image, au grandissement 15, d'une mire de Foucault d'un type spécial, réalisée par les Laboratoires THOMSON-C. S. F. (LCR de Corbeville). Cette mire est fabriquée en déposant sur un substrat de silicium (épaisseur $5 \mu \mathrm{m}$ ) des barreaux

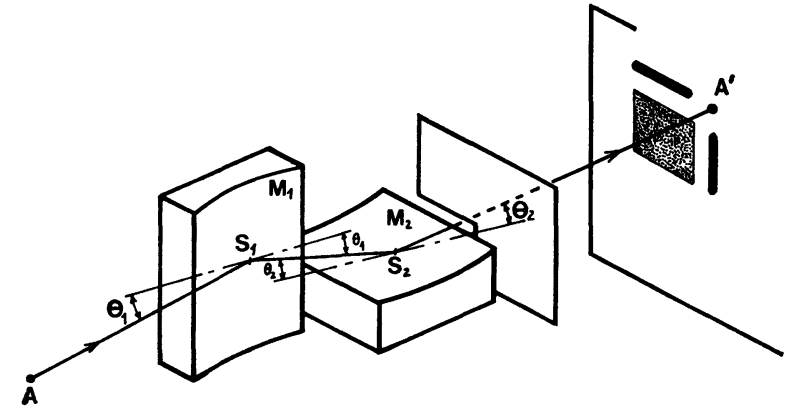

Fig. 2. - Schéma de principe d'un microscope $X$ à 2 miroirs cylindriques (M 1 et $M 2$ ) croisés.

[Principle of an X-ray microscope with two crossed cylindrical mirrors.]

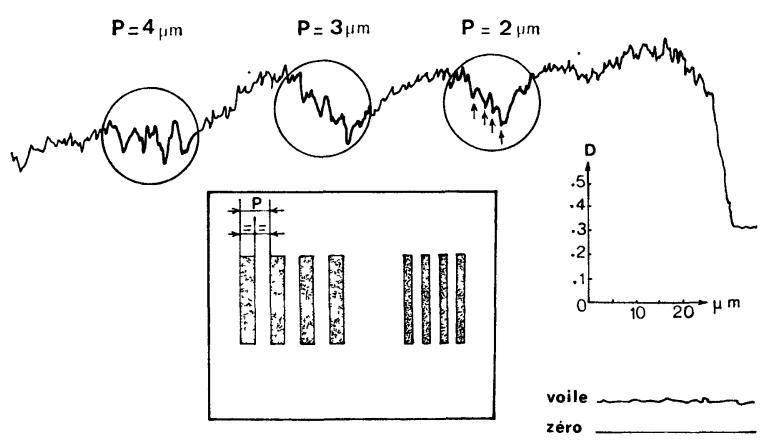

FIG. 3. - Densitogramme de l'image $X(\lambda=3 \AA)$ d'une mire de Foucault.

[Microdensitometer trace of an X-ray picture.]

d'or d'épaisseur $1 \mu \mathrm{m}$; l'intervalle entre chaque barreau est égal à sa largeur qui varie entre 1 et $5 \mu \mathrm{m}$. Pour un rayonnement $X$ de longueur d'onde voisine de $3 \AA$, le contraste image est bien entendu très faible ; néanmoins on distingue les 4 barreaux de la mire de pas $2 \mu \mathrm{m}$.

L'angle d'attaque (complément de l'angle d'incidence) des miroirs étant très faible (environ $10 \mathrm{mrd}$ ), les aberrations sont importantes et limitent rapidement le champ-objet [7]. En particulier, la forte inclinaison de l'image par rapport au rayon moyen du faisceau entraîne une diminution de la résolution lorsqu'on s'en écarte. La limite de résolution est alors proportionnelle à la distance du point observé au rayon moyen et elle est minimale au centre du champ. Elle est aussi proportionnelle à l'ouverture des miroirs. Ainsi, pour obtenir une résolution de valeur donnée, il faut limiter le champ-objet à un cercle centré sur le rayon moyen dont le diamètre est inversement proportionnel à l'ouverture; dans l'exemple présenté ici, ce diamètre est d'environ $20 \mu \mathrm{m}$ pour une résolution spatiale de $2 \mu \mathrm{m}$.

La figure 4 illustre la propriété de filtrage spectral des microscopes; elle représente la variation du coefficient de réflexion de l'or et du verre en fonction. de l'angle d'attaque à la longueur d'onde donnée. 


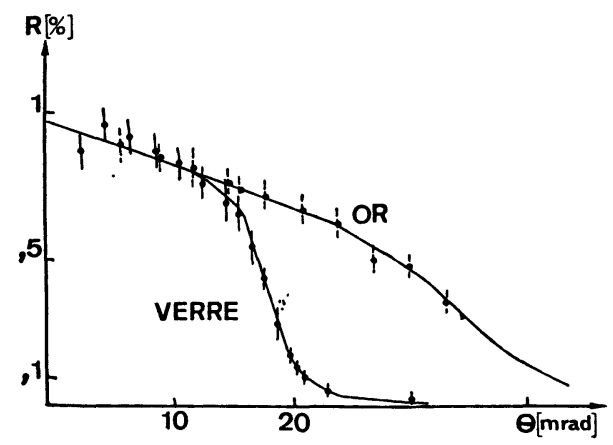

Fig. 4. - Variation du coefficient de réflexion $R$ de l'or et du verre en fonction de l'angle d'attaque $\theta$ (complément de l'angle d'incidence) pour un rayonnement d'énergie $1,742 \mathrm{keV}: \phi$ points expérimentaux ; - courbes théoriques.

[Reflection efficiency $R$ of glass and gold as a function of the grazing incident angle $\theta$ (X-ray energy $1.742 \mathrm{keV}): \phi$ expérimental points ; - theoretical curves.]

Le coefficient de réflexion chute brutalement au voisinage d'un angle d'attaque limite, d'autant plus élevé que le matériau est plus lourd. On peut remarquer le bon accord entre les résultats expérimentaux (mesures réalisées dans nos laboratoires) et théoriques.

Actuellement, pour améliorer le rendement lumineux du système nous envisageons la mise au point d'un microscope du type Wolter dont les tolérances de fabrication sont draconniennes (écart à la forme des surfaces $<500 \AA$ )

3. Récepteurs. - Une étude conduite en collaboration avec la Société LEP [8] a montré que le domaine $\mathrm{X}$ compris entre 1 et $10 \mathrm{keV}$ pouvait être couvert par l'emploi de photocathodes métalliques. Leur temps de réponse est subpicoseconde, leur rendement quantique est relativement élevé (de l'ordre de quelques \% à $10 \%$ ). La figure 5 montre que pour l'or il existe une épaisseur optimale pour laquelle le rendement quantique est maximal dans le domaine $1-10 \mathrm{keV}$; la

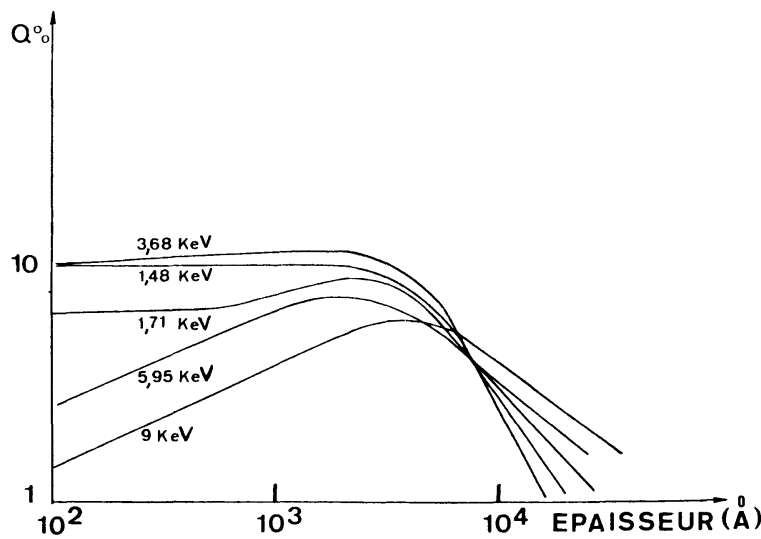

Fig. 5. - Variation du rendement quantique $Q$ d'une photocathode en or en fonction de l'épaisseur et pour différentes énergies $\mathrm{du}$ rayonnement incident.

[Quantum efficiency $Q$ of gold as function of the photocathode thickness at different energies] largeur du spectre en énergie des photoélectrons est environ 100 fois plus élevée que celle observée dans le domaine du rayonnement visible. La figure 6 montre que pour une photocathode en or irradiée par un

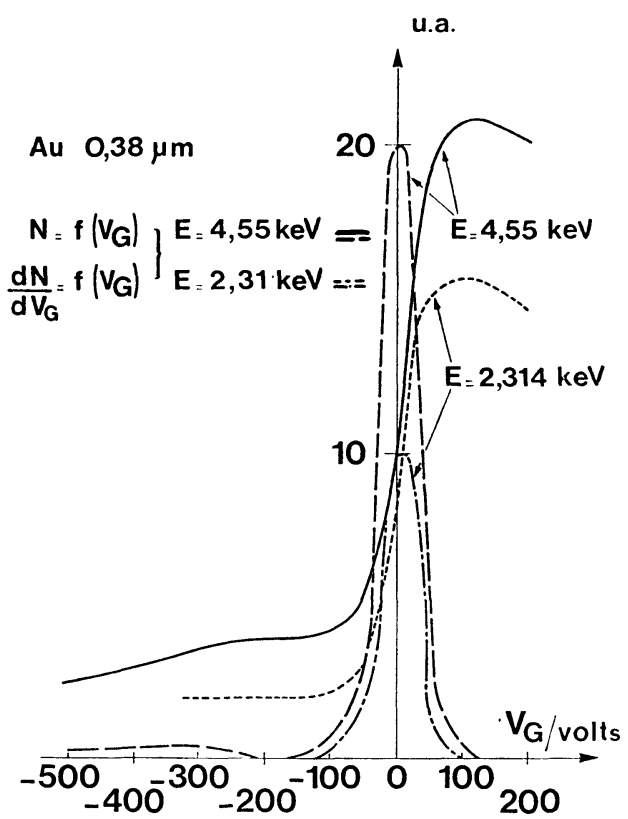

Fig. 6. - Variations du nombre de photoélectrons $N$ en fonction d'un potentiel retardateur $V_{G}$ appliqué entre une photocathode en or (épaisseur $0,38 \mu \mathrm{m}$ ) et une grille pour 2 énergies du rayonnement incident 4,55 et $2,314 \mathrm{keV}$. La répartition énergétique des électrons est représentée par les courbes représentatives de la fonction $\mathrm{d} N / \mathrm{d} V_{\mathrm{G}}=f\left(V_{\mathrm{G}}\right)$.

[Variations of the number of photoelectrons $N$ as a fonction of a retardating voltage $V_{\mathrm{G}}$ applied between a gold photocathode (thickness $0.38 \mu \mathrm{m}$ ) and a grid, for two X-ray energy 4.55 and $2.314 \mathrm{keV}$. Energy distribution of photoelectrons is given by $\mathrm{d} N / \mathrm{d} V_{\mathrm{G}}=f\left(V_{\mathrm{G}}\right)$.]

rayonnement $\mathrm{X}$ d'énergie 2,3 et $4,5 \mathrm{keV}, 90 \%$ des photoélectrons ont une énergie inférieure à $100 \mathrm{eV}$. A cet élargissement spectral correspond une dispersion accrue des temps de transit des photoélectrons entre la photocathode et l'écran de visualisation des caméras. Ce phénomène s'oppose donc à l'obtention de très hautes résolutions temporelles. Heureusement nous pouvons en minimiser l'influence en augmentant le champ d'extraction des photoélectrons au niveau de la photocathode $[9,10]$.

Dans ces conditions nous avons pu réaliser deux types de caméras.

3.1 Tube Obturateur. - Pour obtenir des images en deux dimensions et des temps d'observation de 200 ps, nous utiliserons en 1977 un tube obturateur de conception originale.

En effet nous avons observé qu'en plaquant une photocathode en or sur la face d'entrée d'une galette de microcanaux (multiplicateur d'électrons) on obtenait :

1) Une collimation angulaire des photoélectrons ; 
2) Une réponse spectrale quasi uniforme entre 1 et $10 \mathrm{keV}$ (Fig. 7);

3) Un spectre d'énergie des électrons relativement étroit $(<50 \mathrm{eV})$ à la sortie d'une galette à canaux courbes.

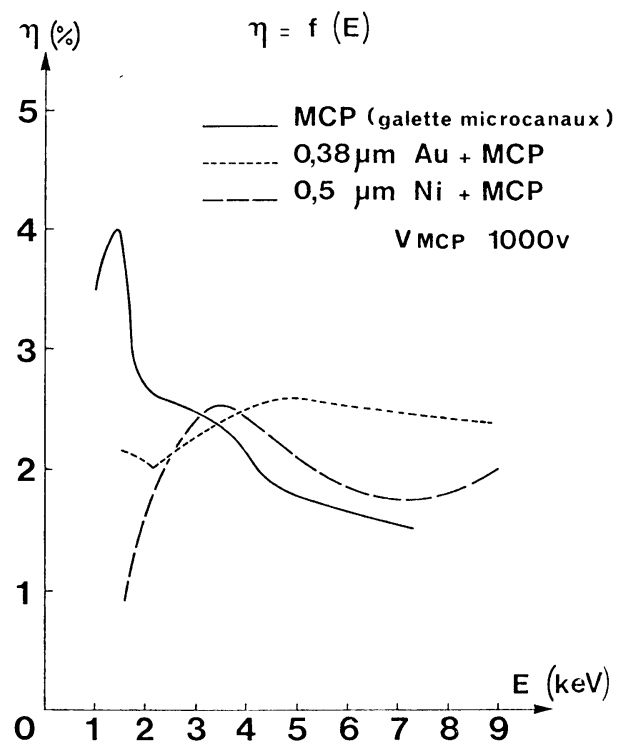

FIG. 7. - Variation du rendement quantique $\eta$ d'une galette de microcanaux (MCP), associée ou non avec une photocathode en or, en fonction de l'énergie $E$ du rayonnement incident.

[Detection efficiency $\eta$ of a microchannel plate associated or otherwise with a gold photocathode as a function of the X-ray energies $E$.]

Dans ces conditions, la possibilité d'obtenir une résolution spatiale de $100 \mu \mathrm{m}$ et un rapport d'obturation $\left(^{*}\right)$ de l'ordre de $10^{5}$ était établie. En ce qui concerne le temps d'exposition de 200 à 300 ps, la solution retenue (Fig. 8) consiste à réaliser une ligne de propagation d'impédance $50 \mathrm{ohms}$, incorporant

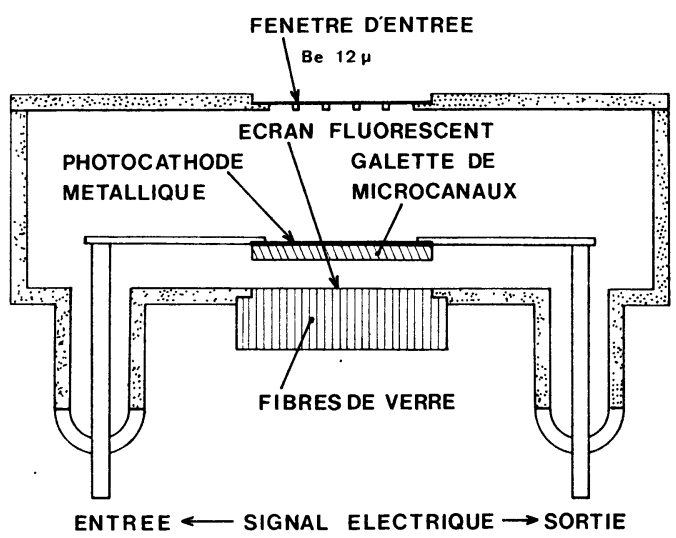

FIG. 8. - Schéma de principe d'un tube obturateur X. [Principle of a shutter tube.]

(*) Nota : le rapport d'obturation est défini par le rapport sntre les quantités de lumière reçues par le film placé derrière le tube lorsque ce dernier est ouvert et lorsqu'il est fermé. à la fois la galette des microcanaux et l'espace écran de visualisation-galette, la galette de microcanaux n'étant pas métallisée sur sa face de sortie.

3.2 Caméras a balayage de fente. - Pour examiner l'évolution temporelle à quelques $10 \mathrm{ps}$ près de l'image du plasma suivant un de ses diamètres, nous utilisons des caméras à balayage de fente. Elles sont identiques à celles que nous utilisons dans le domaine du rayonnement visible, mais le tube convertisseur d'image est équipé d'une photocathode métal-

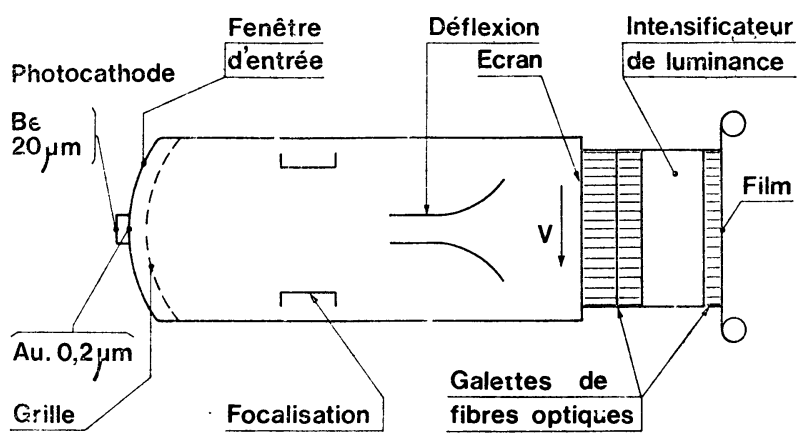

Fig. 9. - Schéma de principe d'un tube $X$ convertisseur d'image pour caméra à balayage de fente.

[Principle of an X-ray streak tube.]

lique [11]. Sur la figure 9 qui rappelle le principe d'une caméra à balayage de fente, on peut observer :

1) que la photocathode est déposée sur une feuille de béryllium mince (d'épaisseur $12 \mu \mathrm{m}$ à $25 \mu \mathrm{m}$ ) transparente aux rayons $X$ jusqu'à $1 \mathrm{keV}$, et assurant l'étanchéité au vide du tube convertisseur d'image ;

2) qu'une grille placée au voisinage de la photocathode (à $5 \mathrm{~mm}$ ) permet d'accroître le champ d'extraction des photoélectrons et donc de diminuer la dispersion du temps de transit de ces derniers entre la photocathode et l'écran de visualisation;

3) que le gain lumineux du tube convertisseur d'image lui-même est accru à l'aide d'un intensificateur de luminance couplé par fibres optiques à l'écran de visualisation.

Dans ces conditions, pour un gain lumineux global de 5000 , un champ d'extraction des photoélectrons de $3500 \mathrm{~V} / \mathrm{cm}$ et une vitesse de balayage de $2 \times 10^{9} \mathrm{~cm} / \mathrm{s}$, les performances obtenues sont les suivantes :

- éclairement minimum détectable $100 \mathrm{~mW} / \mathrm{cm}^{2}$;

- résolution spatiale : $150 \mu \mathrm{m}$;

- résolution temporelle : quelques dizaines de picosecondes ;

— dynamique d'enregistrement : 50 à 100.

La figure 10 montre l'évolution temporelle du flux $X$ émis par un plasma créé par focalisation d'un faisceau laser à verre dopé au néodyme (énergie 2 joules ; durée à mi-puissance $100 \mathrm{ps}$ ) sur une cible d'aluminium. La forme temporelle de cette impulsion $\mathrm{X}$ est 


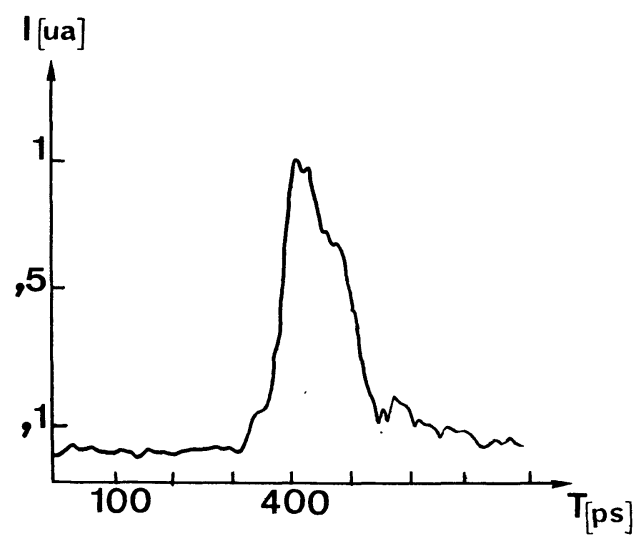

Fig. 10. - Variation temporelle de l'émission $\mathrm{X}$ d'un plasma créé par laser, observée avec une caméra à balayage de fente. [X-ray streak camera : microdensitometer trace of an X-ray pulse produced by laser.]

identique, aux erreurs de mesures près, à celle de l'impulsion laser. Il est donc certain que la résolution temporelle de la caméra $\mathrm{X}$ est inférieure au front de montée de l'impulsion enregistrée, soit 50 ps.

D'autre part la résolution temporelle des caméras $\mathrm{X}$ à balayage de fente peut encore être accrue et atteindre 5 à 10 picosecondes à condition d'accroître :

- le champ d'extraction des photoélectrons jusqu'à une valeur supérieure à $10 \mathrm{kV} / \mathrm{cm}$;

- la vitesse de balayage jusqu'à $10^{10} \mathrm{~cm} / \mathrm{s}$;

- la résolution spatiale ;

- le gain lumineux du tube en lui incorporant, comme cela existe déjà pour les caméras que nous utilisons dans le domaine visible, une galette de microcanaux en amont de l'écran de visualisation.

Néanmoins il est important de rappeler que la résolution spatiale et la résolution temporelle ne sont pas indépendantes. La mesure densitométrique représente l'intensité du phénomène observé dans un certain intervalle de temps et d'espace mais avec une fluctuation qui dépend du nombre de photoélectrons mesuré dans cet intervalle. Autrement dit, un accroissement de résolution temporelle suppose implicitement que l'on ait un éclairement important au niveau de la photocathode donc une densité de courant élevée sans apparition d'un phénomène de charge d'espace soit environ $1 \mathrm{~A} / \mathrm{cm}^{2}$ pour une résolution temporelle de 1 ps. Dans le cas contraire, la résolution spatiale et la dynamique d'enregistrement deviendraient inexistantes.

Les tubes convertisseurs d'image actuels ne semblent pas répondre à ce critère. Par exemple, la figure 11 montre quelques résultats obtenus dans

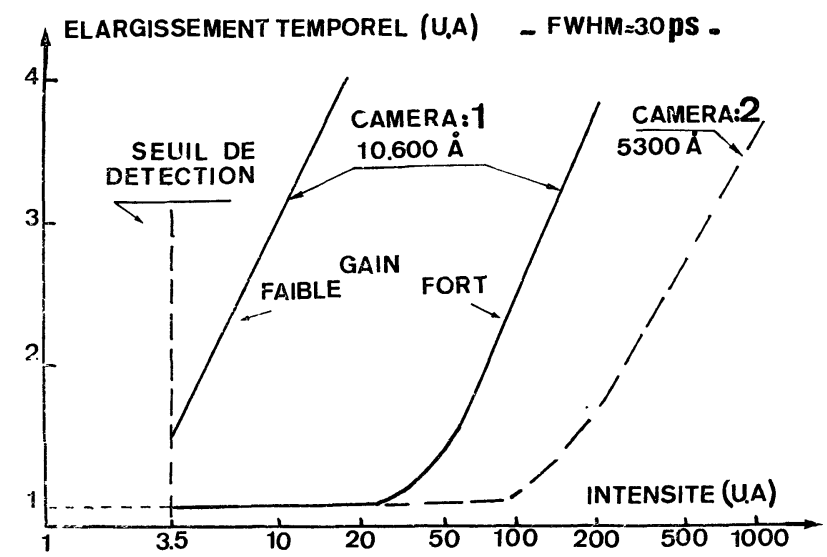

Fig. 11. - Caméra à balayage de fente. Mise en évidence de l'élargissement temporel de l'impulsion laser enregistrée avec l'intensité de rayonnement incident.

[Streak camera : temporal broadening (F. W. H. M.) of the recorded laser pulse with the incident intensity.]

le domaine du rayonnem $\_$nt visible avec deux types de caméras à balayage de fente [12]. Dès que l'éclairement photocathode devient trop important (environ $10^{-2} \mathrm{~A} / \mathrm{cm}^{2}$ ) la largeur à mi-puissance enregistrée, d'une même impulsion, croît. Nous avons également observé ce phénomène dans le domaine $X$.

Nous pensons néanmoins que ce dernier phénomène sera minimisé par l'emploi du nouveau type de tube convertisseur d'image à optique bilamellaire, actuellement en cours de développement à la société LEP.

4. Conclusion. - Les systèmes que nous venons de décrire offrent aujourd'hui des performances spectroet spatio-temporelles extrêmement intéressantes déjà largement mises à profit dans les diagnostics pratiqués autour de nos expériences d'interaction laser-matière et d'impulsions. A moyen terme, une amélioration sensible de ces performances est envisageable.

\section{Bibliographie}

[1] Billon, D., Holstein, P. A., Launspach, J., Patou, C., Reisse, J. M., SchirmanN, D., Laser driven implosion experiments at Limeil. Presented at the Fourth Workshop on laser Interaction and Related Plasma Phenomena. Rensselaer Polytechnic Institute, Troy, New York, Nov. 8-12 (1976).

[2] Holzrichter, J. F. et al., Plasma Phys. 18 (1976) 675.

[3] Seward, F. et al., Rev. Sci. Instrum., 47 (1976) 464.

[4] ChaSe, R. C., Silk, J. K., Appl. Opt. 14 (1975) 2096.

[5] Kirkpatrick, P., Baez, A. V., J. O. S. A. 38 (1948) 766.

[6] Wolter, H., Ann. Phys. 10 (1952) 94 et 286.

[7] Montel, M., Ann. Phys. (1958) 859.
[8] Chalmeton, V., Roux, J. P., Clément, G., Gex, J. P., Advances in $X$ ray analysis, 19. Denver (1975) 507.

[9] Atwood, D. T. et al., Phys. Rev. Lett. 37 (1976) 499.

[10] Bradley, D. J., et al., Opt. Commun. 15 (1975) 231.

[11] Fleurot, N., Gex, J. P., Lamy, M., Quinnessière, C., Sauneuf, R., Caméra $X$ à balayage de fente et haute résolution temporelle avec microscope $X$ associé (même congrès que [12]).

[12] Fleurot, N., GeX, J. P., Bardes, J., Chappet, B., De la difficulté à caractériser une impulsion laser picoseconde à $1060 \mathrm{~nm}$ au moyen de caméras à balayage de fente. Annales du $12^{e}$ Congrès International de Photographie ultra-rapide, Toronto, 1-7 août 1976. 